A broadband high-resolution elliptical crystal $x$-ray spectrometer for high energy density physics experiments

S. G. Anderson, R. F. Heeter, R. Booth, J. Emig, S. Fulkerson, T. McCarville, D. Norman, B. K. F. Young

April 6, 2006

Review of Scientific Instruments 
This document was prepared as an account of work sponsored by an agency of the United States Government. Neither the United States Government nor the University of California nor any of their employees, makes any warranty, express or implied, or assumes any legal liability or responsibility for the accuracy, completeness, or usefulness of any information, apparatus, product, or process disclosed, or represents that its use would not infringe privately owned rights. Reference herein to any specific commercial product, process, or service by trade name, trademark, manufacturer, or otherwise, does not necessarily constitute or imply its endorsement, recommendation, or favoring by the United States Government or the University of California. The views and opinions of authors expressed herein do not necessarily state or reflect those of the United States Government or the University of California, and shall not be used for advertising or product endorsement purposes. 


\title{
A broadband high-resolution elliptical crystal x-ray spectrometer for high energy density physics experiments
}

\author{
S.G. Anderson, ${ }^{*}$ R.F. Heeter, R. Booth, J. Emig, S. Fulkerson, T. McCarville, D. Norman, and B.K.F. Young \\ Lawrence Livermore National Laboratory, \\ 7000 East Ave, Livermore, CA 94550
}

(Dated: March 28, 2006)

\begin{abstract}
Spectroscopic investigation of high temperature laser produced plasmas in general, and x-ray opacity experiments in particular, often requires instruments with both a broad coverage of x-ray energies and high spectral, spatial, and temporal resolution. We analyze the design, model the response, and report the commissioning of a spectrometer using elliptical crystals in conjunction with a large format, gated microchannel plate detector. Measurements taken with this instrument at the JANUS laser facilities demonstrate the designed spectral range of 0.24 to $5.8 \mathrm{keV}$, and spectral resolution $E / \Delta E>500$, resulting in 2 to 3 times more spectral data than achieved by previous spectrometer designs. The observed 100 picosecond temporal resolution and $35 \mu \mathrm{m}$ spatial resolution are consistent with the requirements of high energy density opacity experiments.
\end{abstract}

\section{INTRODUCTION}

X-ray spectroscopy plays a key role in the characterization of hot, dense matter as found in inertial confinement fusion plasmas [1] and stellar interiors [2, 3]. Measurement of the photoabsorption of these plasmas is critical to understanding their behavior, and places stringent demands on the diagnostic to be used. Specifically, the absorption of the plasma must be measured over the broad spectral range relevant to radiation transport, and the spectral resolution must be high enough to distinguish features indicative of the underlying physics. In order to compare with theoretical models, the temperature and density of the plasma must be measured concurrently with the absorption and self-emission spectra, implying a time-gated and spatially resolved measurement [4]. Furthermore, for a precision opacity measurement, a relative intensity calibration accuracy on the order of several percent is desirable.

To meet these experimental requirements, we employ an elliptical crystal geometry [5-7]. In this arrangement the $\mathrm{x}$-ray source is placed at one focus of the ellipse. Xrays reflected by the crystal converge at the other ellipse focus, where a slit is placed to shield the detector from unwanted radiation. In an elliptical geometry, extended sources are demagnified, which reduces source broadening and improves spectral resolution. The instrument discussed here achieves resolving powers comparable with elliptical crystal spectrometers presently in use [8, 9], but has a much greater spectral range. This is achieved using multiple crystal types on geometrically equivalent ellipses rotated slightly about the same major axis for spatial separation, and by using a larger portion of the ellipse, resulting in a greater range of Bragg angles.

The need for high resolution has motivated the development of a new, gated, large format, micro-channel

\footnotetext{
*Electronic address: anderson131@llnl.gov
}

plate (MCP) detector [10]. In this detector, the gate pulse is driven across the short axis of the MCP, perpendicular to the spectral dispersion axis. The result is improved image simultaneity and reduced gain variation along the spectral axis of the MCP.

The spectrometer design, modeling of the integrated spectrometer and detector, and characterization of the instrument performed at the Janus laser facility are described in this paper. Time gated and spatially resolved spectra are used to calibrate and analyze the performance of the instrument.

\section{INSTRUMENT DESIGN}

The application driven spectral coverage requirement of roughly $200 \mathrm{eV}$ to $6000 \mathrm{eV}$ was achieved using three different elliptically bent crystals; octadecyl hydrogen maleate (OHM), rubidium acid phthalate (RAP), and pentaerythritol (PET), with $2 d$ lattice spacings of $63.5 \AA$, $26.121 \AA$, and $8.742 \AA$, respectively. A range of Bragg angles from $14^{\circ}$ to $55^{\circ}$ was chosen to meet the spectral requirements, as well as provide some overlap in coverage of the three crystals. The parameters that define the spectrometer geometry are the ellipse eccentricity $\varepsilon$, focal length $f$, and inclination angle $\eta$, of the semi-major axis with respect to the source-diagnostic port centerline.

The choice of ellipse parameters, and the location of the $10 \mathrm{~cm}$ long MCP detector, was a compromise that maximized spectral resolution while remaining within the physical constraints of the system. These constraints include the diagnostic space envelope at the Omega laser facility, and minimum radius of curvature to which the crystals can be bent. Twenty five millimeter radii have been achieved for PET, RAP, and most recently OHM crystals. A ray-tracing computer script was developed to scan over the $(\varepsilon, f, \eta)$ configuration space [8]. The final design ray-tracing has parameters $\varepsilon=0.97539$, $f=350 \mathrm{~cm}$, and $\eta=1.187^{\circ}$. The locations of the elliptical crystal segment and MCP detector surface in relation to the x-ray source and diagnostic space envelope 


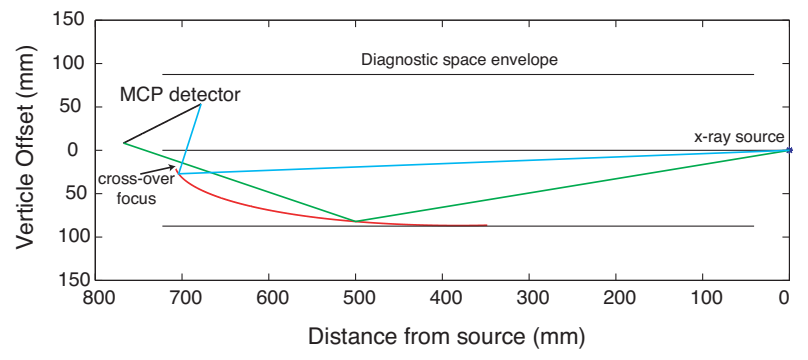

FIG. 1: Geometry of the elliptical crystal spectrometer. The design parameters are: $\varepsilon=0.97539,2 f=700 \mathrm{~cm}$, and $\eta=$ $1.187^{\circ}$

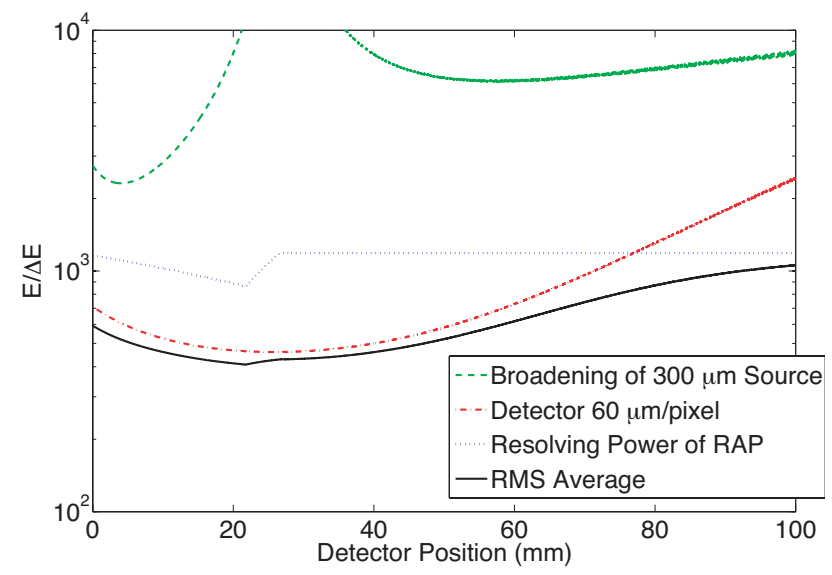

FIG. 2: Effective resolving power of the spectrometer. The effects of source broadening (0.3 mm source), detector spatial resolution (60 $\mu \mathrm{m}$ effective pixel size), and the RAP crystal resolving power [11] are included.

are shown schematically in Fig. 1.

The overall spectral resolving power of the instrument is estimated by summing in squares the resolving powers due to the various limiting components of the system. The net resolving power is plotted in Fig. 2, with the resolution limits due to spectral broadening of a $300 \mu \mathrm{m}$ source, the $60 \mu \mathrm{m}$ effective spatial resolution of the MCP, and the inherent resolving power of an elliptically bent RAP crystal [11]. The resolving power limit due to spatial resolution of the detector was calculated using the assumption that a minimum of 3 detector 'pixels' are required for a full width at half maximum (FWHM) line width measurement. As Fig. 2 shows, detector resolution limits the resolving power of the instrument over most of its spectral range, with crystal inherent energy resolution becoming important at low energies (larger detector positions). The predicted resolving power of the instrument, $E / \Delta E \geq 400$ over the full spectral range.

The instrument sensitivity has been modeled to aid in the choice of filters for each crystal, and to provide a calibration of relative x-ray intensity of measured data. While the inclusion of film response, filter transmission, and crystal reflectivities [6] are straight-forward, an accurate model of the MCP response is more complicated.

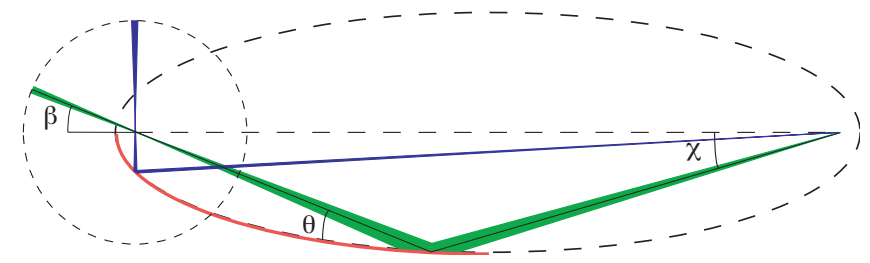

FIG. 3: Defining angles in the elliptical geometry.

The detection of x-rays by MCPs has been studied both theoretically and experimentally [12-14]. Our analysis closely follows that of Landen et al. (Ref. [14]), which has demonstrated good agreement with experimental observations.

The response per unit area, $Q$, of the $\mathrm{MCP}$ is a function of x-ray incidence angle, $\theta$, energy, $E$, and MCP material. It can be expressed as

$Q \sim S(E)\left[\frac{R(E)}{\operatorname{mfp}(E) \sin \theta}\right] \cos \theta \sum_{n}^{\# \text { pores }} \int_{\text {pore }} I_{n}(x) G_{n}(x) d x$

where $S$ is the number of secondary electrons produced per photoelectron, $R$ is the photoelectron range in the channel plate material, mfp is the x-ray mean free path, and $I_{n}$ and $G_{n}$ are the x-ray intensity and MCP gain of the $n$th traversed pore. The response is summed over all pores with which the x-ray may interact, and integrated over the possible paths $(x)$ in each pore. $I_{n}$ depends on the attenuation length, $l_{\text {atten }}$, of previous pores according to

$$
I_{n}(x)=I_{0} \exp \left(-\frac{l_{\text {atten }}(\theta, n, x)}{\operatorname{mfp}(E)}\right),
$$

and $G_{n}$ is given by the discrete dynode gain model as

$$
G_{n}(x)=\left(\frac{V}{V_{0}}\right)^{\frac{L}{4 D}\left(1-\frac{x}{L}\right)},
$$

where $L$ and $D$ are the pore length and diameter, respectively. The MCP employed here has $D=10 \mu \mathrm{m}$, and $L / D=40$ [10]. The $\cot (\theta)$ dependence of MCP sensitivity, indicated by Eq. 1 and experimentally verified in Ref. [14], motivated the choice of detector orientation to minimize the average $\mathrm{x}$-ray incidence angle.

For elliptical designs, geometric considerations play an important role in the instrument response. Following Ref. [5], we define three angles that characterize this geometry, as shown in figure 3 . Here $\chi$ is the angle from a point source to a given differential element of the ellipse measured from the semi-major axis, $\theta$ is the Bragg angle of the ray with the ellipse, and $\beta$ is the angle made by the reflected ray passing through the cross-over focus of the ellipse. These angles follow the relation,

$$
\chi=2 \theta-\beta .
$$

To calculate the number of photons per second per unit area at the detector we define two length parameters, $r$, 


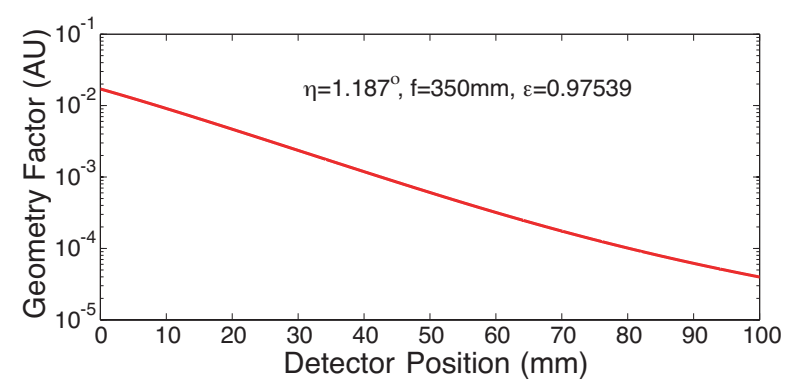

FIG. 4: Geometry factor, $\mathcal{F}_{g}$, influencing the x-ray intensity reaching the detector versus detector position in the OZSPEC design.

the distance from the cross-over focus of the ellipse to the detector, and $L$, the distance from the source to the ellipse element added to the distance from the ellipse to the detector. In addition, we assume that the diffracting crystal has a small extension normal to the plane of the ellipse, and define an angle $\psi$, such that the differential solid angle from the source of the diffracted beam is $\psi d \chi$. A differential area of detector is then given by $d A=$ $r d \beta L \psi$.

The number of photons reflected from a differential length of crystal is found from the integrated reflectivity, $R$, the area under the reflectivity curve, $I\left(\theta-\theta_{\text {Bragg }}\right)$. Given these definitions, and assuming a source with intensity $S$ photons/steradian-second-eV at photon energy $E$, the number of photons per unit area per second at the detector is

$$
\begin{aligned}
\frac{d N}{d A} & =\frac{S}{r L}\left(\frac{d \chi}{d \theta}\right)\left(\frac{d \theta}{d \beta}\right)\left(\frac{d E}{d \theta}\right) R \\
& =S \mathcal{F}_{g} R,
\end{aligned}
$$

where the factor $\mathcal{F}_{g}$ depends only on the geometry of the spectrometer. This factor is easily modified in the case considered here to account for the fact that we use a flat detector surface instead of a circular arc, and is plotted in figure 4 for the OZSPEC design parameters. As the figure shows, the geometry factor has a significant affect, decreasing the photon intensity by over two orders of magnitude from the high to low energy (higher detector position) ends of the channel plate. This affect is mitigated partially by the detector response versus energy, and in many cases, by the spectrum of the source.

The relative response was calculated using the effects discussed above for each crystal and a number of filter materials. Figure 5 shows an example response calculation using an RAP crystal with x-rays filtered by $1 \mu \mathrm{m}$ of Aluminum and $0.1 \mu \mathrm{m}$ of Copper.

\section{MEASUREMENTS}

The spectrometer was commissioned at the JANUS laser facility at LLNL. X-rays were generated with various targets using up to $200 \mathrm{~J}$ of $532 \mathrm{~nm}$ laser light with

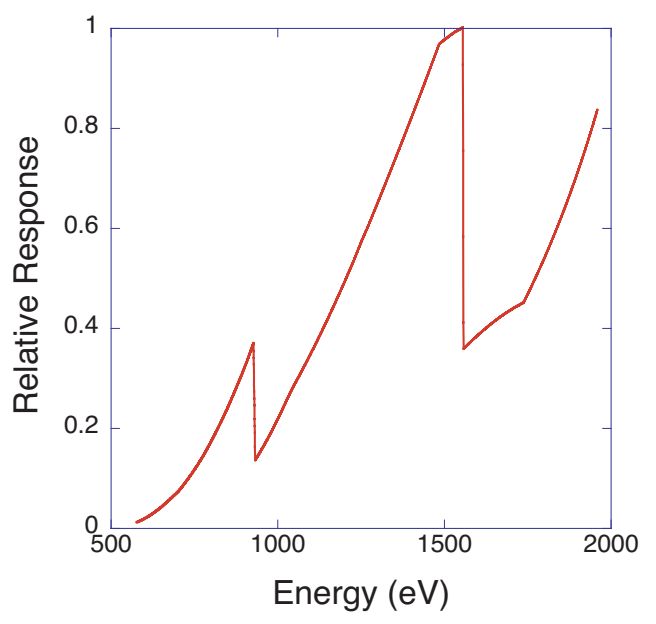

FIG. 5: Calculated relative response of the instrument with an RAP crystal and $\mathrm{Cu}$ and $\mathrm{Al}$ filtering.

a duration of 1-3 ns and a focused spot size of approximately $100 \mu \mathrm{m}$. Target materials were chosen to produce $\mathrm{K}$-shell and L-shell emission lines in the spectral ranges of the three crystals. Spatial resolution in the vertical plane is provided by horizontal slits positioned in the nose of the instrument to give $6 \times$ magnification of the source.

To test spatial resolution, the targets were composed of a substrate material and a horizontally aligned thin foil. The targets were then shot at the interface of the two materials. The previously measured [10] 100 ps detector gain gate pulse was timed to the x-ray pulse arrival using a photoconductive detector (PCD). Filters located between the imaging slits and the crystals were needed to block laser light, and were chosen to produce $\mathrm{K}$ and L-shell absorption edges as a further wavelength scale calibration. A positively biased, phosphor coated fiberoptic plate converts the MCP signal to a light pulse and transmits it to $35 \mathrm{~mm}$ film (TMAX 3200). Film response is removed using standard 4-decade logarithmic gradient wedges co-developed with the film.

Figure 6 shows a digitized image of the film generated by shooting a $\mathrm{SiO}_{2}$ (Pyrex) substrate with a titanium foil. The film shows three spectra corresponding to the three vertically stacked crystals. The spectra is gated, with the gate pulse propagating perpendicular to the spectral dispersion axis (vertically, from bottom to top in Fig 6), with the PET data taken 200 ps before the RAP data. Lineouts of the data were taken, and detector position converted to photon energy using several identified lines and the instrument design model.

Evidence of spatial resolution is shown in Fig. 7, in which a portion of the RAP data of Fig. 6 is analyzed. The vertically separated $\mathrm{Ti}$ and Pyrex plasmas produce separate spectra that are distinguished by taking lineouts through the top and bottom halves of the data. The top lineout (imaging the bottom half of the target, the $\mathrm{Ti}$ foil) shows a spectrum clearly contrasting with the bottom lineout. Lithium-like Titanium lines are identified 


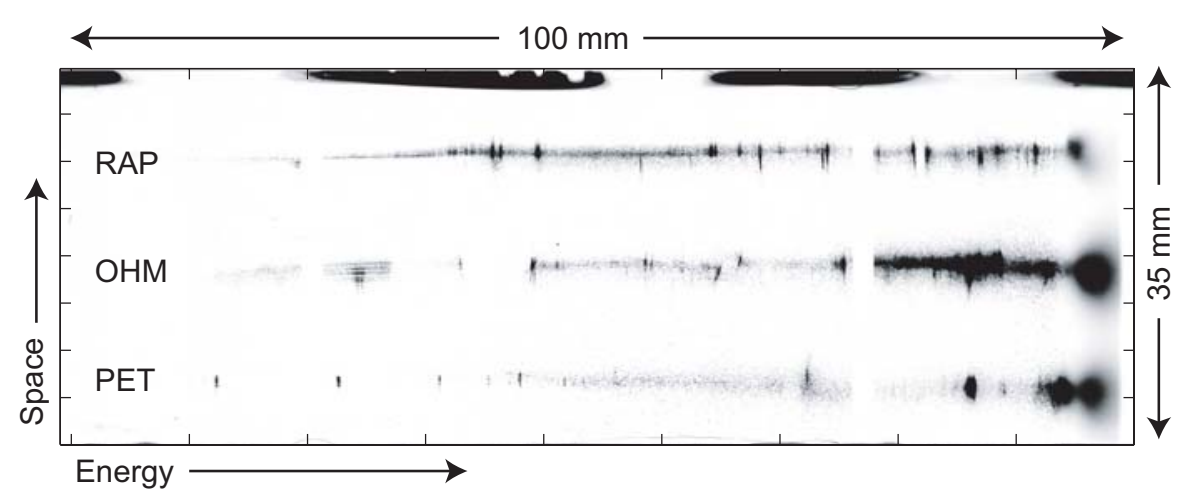

FIG. 6: Image of film recording Pyrex and Titanium target shot at JANUS. The PET (bottom), OHM (middle) and RAP (top) crystals combined capture $250-5500 \mathrm{eV}$ x-rays.
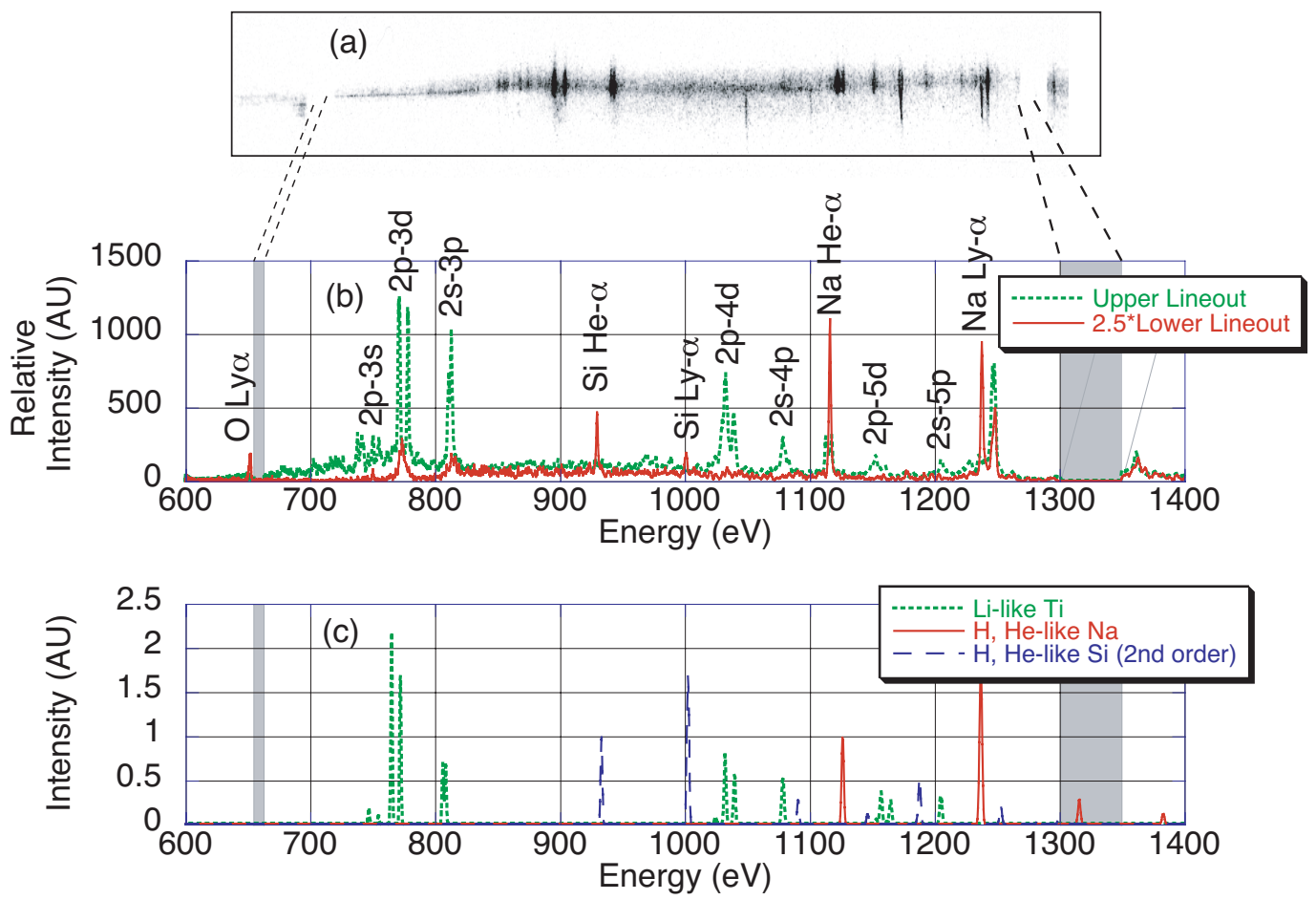

FIG. 7: (a) Enlargement of a section of the RAP spectrum shown in Fig 6. Two sets of horizontal lines indicate the area over which lineouts were taken. (b) Lineouts of the film image in (a). The dotted line is taken from the upper part of the image and shows L-shell transitions of Li-like Ti. The solid line is taken from the lower part of the image and shows O, Na, and Si K-shell lines (the $\mathrm{Si}$ features in $2^{\text {nd }}$ order). (c) FAC [15] generated $\mathrm{Ti}, \mathrm{Si}$, and $\mathrm{Na}$ transition energies matching the lines identified in (b).

on one spectrum, while Hydrogen and Helium-like lines of Oxygen, Sodium, and Silicon (Pyrex constituents) are visible on the other spectrum, with the Si lines in $2^{\text {nd }}$ order. The spatial resolution was quantified by examining lineouts in the vertical direction. The intensity level was found to transition from the Ti to the Pyrex plasmas within $70 \mu \mathrm{m}$, consistent with the use of a $60 \mu \mathrm{m}$ slit and $6 \times$ pinhole imaging. Thirty micron slits have also been employed, yielding $35 \mu \mathrm{m}$ spatial resolution.
Features in the PET and OHM spectra were also identified. These include $\mathrm{K}$-shell lines of $\mathrm{Si}$ and $\mathrm{Ti}$ on the PET crystal and the carbon K-edge of the plastic filter used with the OHM crystal. The full-width at half maximums (FWHM) of several lines on each of the three crystals were measured to determine the spectral resolving power, $E / \Delta E_{\mathrm{FWHM}}$. Values of 500-600 were found for lines on the low to middle energy ranges of each crystal, while lower values, $\approx 300$ were measured at the high 
energy ends.

Gaps in spectral coverage are evident in Figs. 6 and 7. There are two causes of voids. The first cause is a set of 2 mm gaps between sections of the MCP located roughly 25 and $75 \mathrm{~mm}$ from one edge of the detector. The detector surface is divided into three sections in order to facilitate timing adjustments and support a large detector area. The missing portions of spectra caused by MCP inactive channels are indicated by gray bands in Fig. 7 and in Fig. 8, which shows the complete spectrum from Fig. 6 using all three crystals.

The second cause of losses in spectral coverage comes from imperfect interfaces between segments of crystal on the ellipse surfaces. Tiling multiple crystal pieces along the ellipse is necessary due to lengths of commercially available crystals ( $3 \mathrm{~cm}$ strips in the case of OHM, compared to over $21 \mathrm{~cm}$ of ellipse arc length). The positions of the crystal segment edges were measured and found to agree well with observed irregularities in the spectra. These gaps are indicated in Fig. 8 with cross- hatched areas. The problem has been mitigated in other experiments by using more than one ellipse with the same crystal type, but with offset tiling to produce continuous spectral coverage.

\section{CONCLUSIONS}

A broadband moderately high-resolution elliptical crystal x-ray spectrometer has been fielded at the JANUS laser facility. The spectral range and resolution, and the spatial and temporal resolution are suitable for planned opacity and other high energy density physics experiments on various lasers.

The instrument may be improved in the future by incorporating flat-field detector measurements into the instrument response model, and by adjusting crystal placement to ensure continuous coverage of spectral ranges important to specific experiments.
[1] B. Yaakobi, D. Steel, E. Thorsos, A. Hauer, and B. Perry, Phys. Rev. Lett. 39, 1526 (1977).

[2] L. B. D. Silva, B. J. MacGowan, D. R. Kania, B. A. Hammel, C. A. Back, E. Hsieh, R. Doyas, C. A. Iglesias, F. J. Rogers, and R. W. Lee, Phys. Rev. Lett. 69, 438 (1992).

[3] P. T. Springer, D. J. Fields, B. G. Wilson, J. K. Nash, W. H. Goldstein, C. A. Iglesias, F. J. Rogers, J. K. Swenson, M. H. Chen, A. Bar-Shalom, et al., Phys. Rev. Lett. 69, 3735 (1992).

[4] B. K. Young, M. B. Schneider, R. F. Heeter, and P. T. Springer, in Proceedings of the JOWOG (Joint Working Group) 37 (2002).

[5] B. L. Henke, H. T. Yamada, and T. J. Tanaka, Rev. Sci. Instrum. 54, 1311 (1983).

[6] B. L. Henke and P. A. Jaanimagi, Rev. Sci. Instrum. 56, 1537 (1985).

[7] B. A. Hammel, D. W. Phillion, and L. E. Ruggles, Review of Scientific Instruments 61, 1920 (1990), URL http: //link.aip.org/link/?RSI/61/1920/1.
[8] R. F. Heeter, J. A. Emig, K. B. Fournier, S. B. Hansen, M. J. May, and B. K. F. Young, Rev. Sci. Instrum. 75, 3762 (2004).

[9] P. W. Lake, J. E. Bailey, G. A. Rochau, T. C. Moore, D. Petmecky, and P. Gard, Rev. Sci. Instrum. 75, 3690 (2004).

[10] T. McCarville, S. Fulkerson, R. Booth, J. Emig, B. Young, S. Anderson, and B. Heeter, Rev. Sci. Instrum. 76, 103501 (2005).

[11] B. L. Henke, E. M. Gullikson, and J. C. Davis, At. Data Nucl. Data Tables 54, 181 (1993).

[12] J. E. Bateman, Nucl. Instrum. Meth. 144, 537 (1977).

[13] N. Yamaguchi, T. Cho, T. Kondoh, M. Hirata, S. Miyoshi, S. Aoki, H. Maezawa, and Y. Satow, Rev. Sci. Instrum. 60, 2307 (1989).

[14] O. L. Landen, A. Lobban, T. Tutt, P. M. Bell, R. Costa, D. R. Hargrove, and F. Ze, Rev. Sci. Instrum. 72, 709 (2001).

[15] M. F. Gu, ApJ 582, 1241 (2003). 

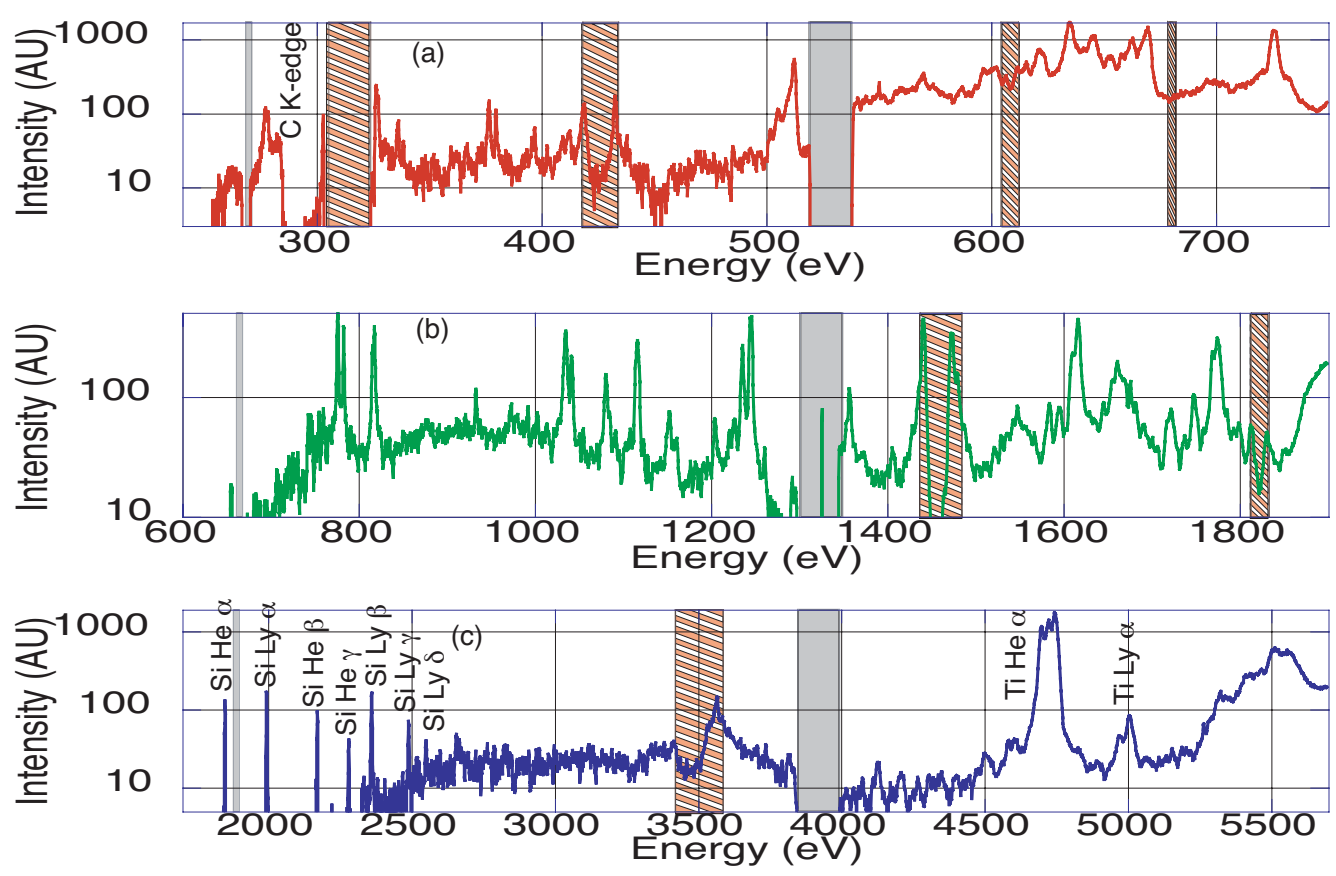

FIG. 8: Spectrum of a pyrex and titanium target derived from the film image in Fig. 6. Lineouts from the OHM (a), RAP (b), and PET (c) crystals show spectral coverage from $250 \mathrm{eV}$ to $5500 \mathrm{eV}$. 